\title{
Looking for trouble: Reduced myocardial flow reserve following anthracyclines
}

\author{
M. C. Ziadi, MD, ${ }^{a}$ Rob de Kemp, PhD, ${ }^{b}$ Rob S. B. Beanlands, MD, FRCPC, FASNC, ${ }^{b}$ \\ and G. R. Small, PhD, MRCPb \\ a Non Invasive Cardiovascular Imaging Department, Instituto Cardiovascular de Rosario, \\ Rosario, Santa Fe, Argentina \\ b Division of Cardiology, Department of Medicine, University of Ottawa Heart Institute, Ottawa, \\ ON, Canada
}

Received Nov 19, 2018; accepted Nov 20, 2018

doi: $10.1007 / \mathrm{s} 12350-018-01564-0$

\section{See related article, pp. 1698-1707}

\section{INTRODUCTION}

Cardio-oncology is an emerging field that addresses cardiovascular diseases in cancer patients. ${ }^{1}$ A central aspect of cardio-oncology practice is the management of cancer therapy-related cardiovascular toxicity. Cancer treatment-related cardiotoxicity can include coronary vasospasm, endothelial dysfunction, myocardial infarction, left ventricular (LV) dysfunction and congestive heart failure (CHF). ${ }^{2}$ When such complications occur, a joint approach is often sought involving cardiology and oncology to navigate a therapeutic course that maintains cancer care and addresses the cardiac concerns. A common source for discussion at cardio-oncology forums is the cardiotoxicity of anthracyclines.

\section{ANTHRACYCLINE CARDIOTOXICITY}

Following their discovery in the 1950s and 1960s, anthracyclines such as doxorubicin became quickly recognized as highly effective, broad-spectrum antineoplastic agents. Reports of cardiotoxicity also

\footnotetext{
M. C. Ziadi and G. R. Small were co-primary authors of this work. Reprint requests: M. C. Ziadi, MD, Non Invasive Cardiovascular Imaging Department, Instituto Cardiovascular de Rosario, 440 Oroño Boulevard, Rosario, Santa Fe, Argentina; mcziadi@hotmail.com

J Nucl Cardiol 2020;27:1708-13.

$1071-3581 / \$ 34.00$

Copyright (c) 2019 American Society of Nuclear Cardiology.
}

surfaced soon after clinical use began. Anthracyclines inhibit tumor DNA and RNA synthesis by intercalating between base pairs and inhibiting the activity of topoisomerase IIa, thus preventing DNA repair. ${ }^{3}$ Anthracycline metabolism within cells may also give rise to free radical production. Free radicals lead to further DNA damage, mitochondrial injury and generation of additional free radicals which disrupts cellular calcium homeostasis and causes cell apoptosis. ${ }^{4}$ Associated acute clinical manifestations may comprise of non-specific repolarization changes on the electrocardiogram, arrhythmias, a myo-pericarditis like picture with troponin elevation, and LV dysfunction. ${ }^{5}$ These changes may be transient and resolve spontaneously following withdrawal of the chemotherapy. In some cases, however, they can also be progressive and lifethreatening (Figure 1). ${ }^{6}$

Anthracycline toxicity may also occur acutely, immediately after the infusion in $<1 \%$ of patients. Alternatively, cardiotoxicity may be seen in $1.6-2.1 \%$ of individuals early during the chemotherapy course or shortly upon completion, or it may occur as a latent effect in cancer survivors (seen in 1.6-5.1\%).,

\section{ASSESSMENT OF CANCER THERAPY-RELATED CARDIAC DYSFUNCTION}

LV ejection fraction (LVEF) is the most widely used measure to detect anthracycline-induced cardiac dysfunction. Current guidelines recommend close monitoring of this parameter and modification or discontinuation of therapy with initiation of appropriate medical interventions at the appearance of LV dysfunction. ${ }^{9}$ Several noninvasive imaging modalities can measure LV performance, including radionuclide angiography (RNA), echocardiography (echo), cardiac 


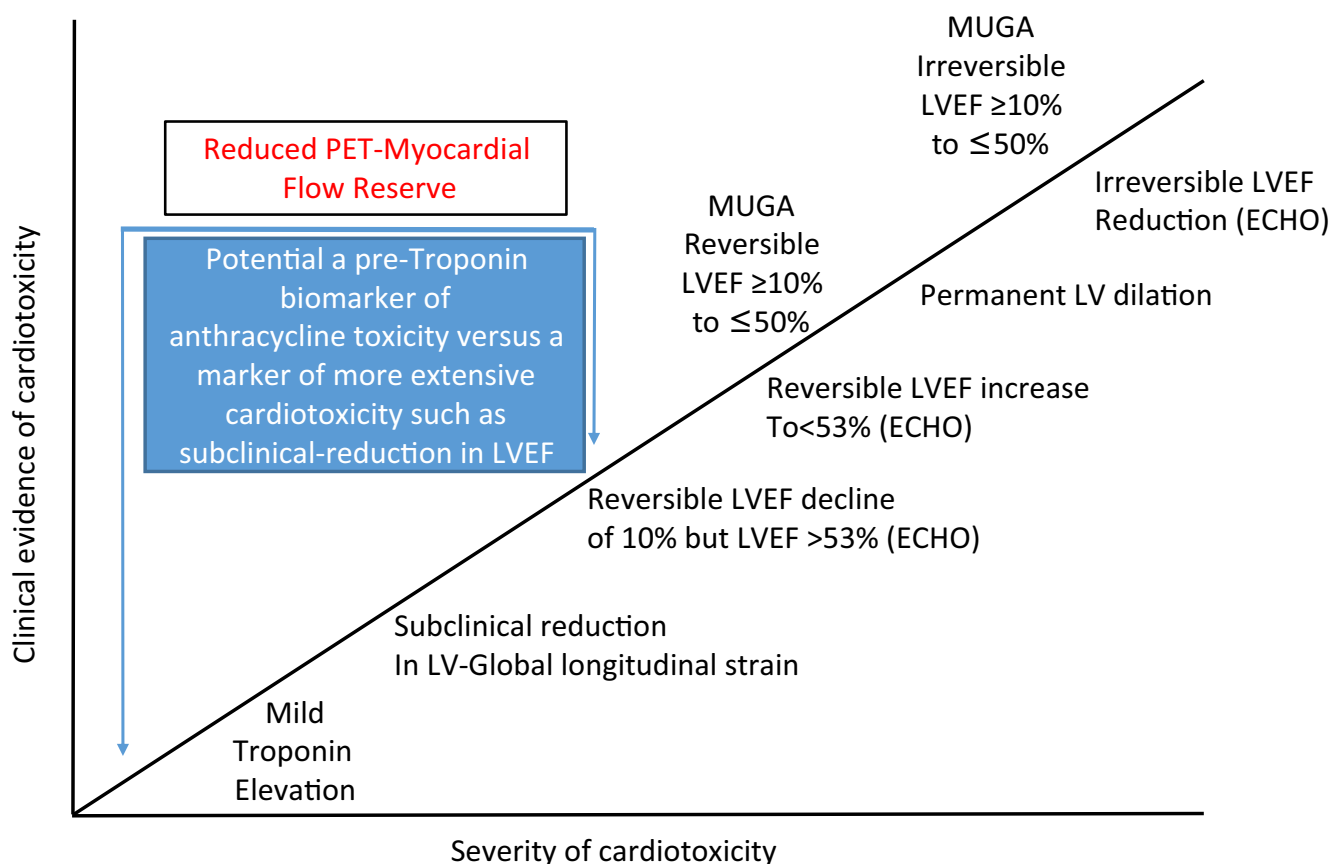

Figure 1. Schematic graph of the severity of cardiotoxicity in terms of clinical biomarkers. Increasing cardiotoxicity is associated with different biomarker findings. Troponin and echocardiography derived global longitudinal strain being markers of preclinical cardiotoxicity. RNA and Echo derived LVEF estimates may be reversible or irreversible. The findings by Laursen have not yet been linked to LVEF decline and are likely subclinical. Whether they are more sensitive than Troponin I, global longitudinal strain or preclinical reductions in LVEF is yet to be determined. ${ }^{19}$ Echo, Echocardiography; $R N A$, radionuclide angiography; $L V E F$, left ventricular ejection fraction.

computed tomography (CT), and cardiac magnetic resonance imaging (CMR). ${ }^{10-13} 2 \mathrm{D}$ Echo is the most widely used but limited by low sensitivity and reproducibility. 3D echocardiography and speckle tracking2D strain echo have been proposed as alternatives, but uptake may vary depending on local availability and expertise. Similarly, cardiac MRI may provide more reproducible measurements of LVEF but its utility is compromised by limited access. RNA studies have the advantage of widespread availability, lower inter-observer variability, high accuracy and reproducibility compared with conventional two-dimensional echocardiography. ${ }^{10,14-16}$ RNA, however, has not been shown to be useful at detecting sub-clinical reductions in LV function which is an important determinant of future clinical cardiotoxicity that can impact ongoing therapy decisions.

\section{CARDIAC PET AND CARDIAC-ONCOLOGY}

Cardiac positron emission tomography (PET) has been investigated as a potentially useful modality to detect cardiac dysfunction or injury following chemotherapy. Prior investigations have considered metabolic, inflammatory and perfusion PET tracers. ${ }^{17,18}$ In this edition of the Journal of Nuclear Cardiology, Laursen et al. suggest that quantitative myocardial blood flow reserve could be a novel method to detect those at risk of anthracycline cardiotoxicity. ${ }^{19}$

\section{MYOCARDIAL FLOW RESERVE}

Myocardial flow reserve (MFR) is the ratio of stress flow to rest flow and represents the capacity of a given coronary bed to maximize flow. It is accurately quantified using PET imaging of rest and pharmacological stress myocardial perfusion tracer kinetics. MFR is incremental to relative perfusion imaging for the investigation of coronary artery disease. ${ }^{20-22}$ MFR may also be abnormal in the absence of epicardial coronary stenosis. This has been described in patients with diabetes and in patients with hypertrophic cardiomyopathy where endothelial dysfunction is disrupted by intimal metabolic changes or abnormal trans-myocardial perfusion gradients, respectively. MFR is also reduced acutely in Takotsubo's cardiomyopathy in association with an inflammatory cell myocardial infiltrate. In Takotsubo's cardiomyopathy the MFR decline usually 
normalizes within 3 months. ${ }^{23}$ MFR represents a sensitive marker of vascular function that may be useful as a marker of early myocardial injury. ${ }^{24}$

\section{MYOCARDIAL BLOOD FLOW AND ANTHRACYCLINES}

Laursen et al. prospectively assessed the role of quantitative perfusion with $82 \mathrm{Rb}$-PET in 54 adult patients diagnosed with lymphoma before and soon after (median time $=3$ days) receiving doxorubicin (presumed dose of $50 \mathrm{mg} / \mathrm{m}^{2}$ ). They identified patients ( $n=13$ out of $54,24 \%$ ) with a decrease in MFR (more than $20 \%$ between baseline and post-exposure PETpreviously defined by KItkivingan test-retest repeatability studies as a meaningful difference.) and classified them as having a low cardiotoxicity threshold (LCT). The MFR changes were independent of the summed stress score (SSS), summed rest score (SRS), summed difference score (SDS) and the coronary artery calcium score. The remainder of the population were categorized as having a high cardiotoxic threshold (HCT). Rest myocardial blood flow tended to increase in patients classified as LCT but did not differ statistically between those with or without LCT. It was the reduction in stress flow in those with LCT that appeared to have important impact on the reduction in MFR. Corrections in rest flow for the rate pressure product did not alter whether patients were identified as LCT or not. In summary, the investigators identified a group of patients who had an acute reduction in stress flow and myocardial flow reserve in response to adenosine following anthracycline administration. This group was older (median 67 years vs 57 years, $\mathrm{p}=0.04$ ). Age is known to be a risk factor for anthracycline cardiotoxicity. Whether the flow response is independent of age as a predictor was not assessed. Defining this group as truly having a low threshold for anthracycline-related cardiotoxicity is also premature at this stage. Nonetheless, there does appear to be a group of patients who have an early abnormal coronary vascular response to anthracycline. This warrants further study.

\section{ANTHRACYCLINE CARDIOTOXICITY AND PET MYOCARDIAL BLOOD FLOW}

Previously, PET-assessed cardiac perfusion has been proposed as a method to predict anthracycline cardiotoxicity. Nony et al investigated whether resting blood flow was affected by anthracyclines by evaluating C11-acetate uptake using PET. ${ }^{18}$ In six patients, blood flow was measured at baseline, 24 hours post doxorubicin (at $50 \mathrm{mg} / \mathrm{m}^{2}$ to a cumulative dose of $300 \mathrm{mg} / \mathrm{m}^{2}$ ) and at 3 weeks after the final dose. Five patients also received cyclophosphamide and 5-fluoro-uracil (both at doses of $500 \mathrm{mg} / \mathrm{m}^{2}$ ). Following the first dose of anthracyclines they observed no difference in resting myocardial blood flow (MBF) following correction for rate pressure product. Similarly, no changes in resting $\mathrm{MBF}$ was seen at the end of the therapy.

The influence of doxorubicin on MFR has been previously considered in a preclinical model of anthracycline cardiotoxicity. ${ }^{25} \mathrm{C} 11$ acetate PET rest and stress perfusion was examined in rats exposed to cardiotoxic anthracycline doses versus control. In comparison to control animals (naïve to anthracycline exposure), doxorubicin-treated animals had lower myocardial blood flow reserve and a lower reserve of myocardial oxygen consumption (calculated as the ratio of stress versus rest myocardial oxygen consumption). LVEF was also significantly reduced in the doxorubicin-treated rats $(38 \%)$ in comparison to controls $(58 \%)$. Although these findings may seem to correlate with those of Laursen et al., the doses of doxorubicin were different to those used clinically. Typical animal models of anthracycline toxicity induce heart failure in all recipients through supra-therapeutic dosing. Such uniformly cardiotoxic doses are not used clinically and so direct extrapolation of these study findings into a clinical setting may not be inappropriate.

\section{CLINICAL UTILITY OF R82 PET FLOW RESERVE IN ANTHRACYCLINE RECIPIENTS}

Application of the findings from Laursen et al. into clinical practice requires further studies. In particular, there are three areas where more data are required. First, more justification is required for defining patients with reduced flow reserve as having a low cardiotoxicity threshold. Other biomarker correlations of subclinical cardiotoxicity were not measured in the study. Agreement is, therefore, required with tests such as highly sensitive troponin or echo strain imaging to confirm that PET flow reserve reduction is a herald of potential cardiotoxicity.

Secondly, in those patients identified as having a high cardiotoxicity threshold what is the incidence of subsequent subclinical or clinical cardiotoxicity? If the preservation of flow reserve is a good prognostic marker for the absence of cardiotoxicity this might be useful to define in patients at high risk from anthracycline cardiotoxicity such as the elderly, those with prior anthracycline exposure or those with prior cardiomyopathy. Follow-up data would be useful.

Finally, in the absence of end point findings such as LVEF, LV global longitudinal strain or troponin results 
or adverse outcomes, it is difficult to determine where the changes in MBF occur in the spectrum of cardiotoxicity. If cancer therapy-related cardiac dysfunction exists as a continuum similar to the ischemia cascade (Figure 1), highly sensitive Troponin might be an early indicator of subclinical cardiotoxicity. Reduced LV global longitudinal strain would likely be close to Tn I as a subclinical marker of myocardial chemotherapy injury. Progressing to more advanced cardiotoxicity would be identified as reversible LV ejection fraction impairment through to LV dilation and permanent LV dysfunction. Whether early MBF reduction is a subclinical herald of pending cardiotoxicity or indicative of more permanent damage needs to be elucidated in longer-term prospective studies, to better understand the potential clinical utility of these findings.

\section{OTHER PET BIOMARKERS OF ANTHRACYCLINE CARDIOTOXICITY HAVE BEEN PROPOSED}

Bauckneht et al and others have considered the observation that in contrast to those patients without cardiac dysfunction, 18F-FDG myocardial uptake is increased in patients that develop anthracycline cardiotoxicity. ${ }^{26}$ The impression from these observations is that whether via an inflammatory response or by induction of myocardial metabolism, anthracyclines induce FDG uptake to a greater degree in those patients who demonstrate cardiotoxicity.

The PET tracer C11-acetate may be used to detect changes in mitochondrial function that could be relevant in anthracycline cardiotoxicity. During cellular respiration in the mitochondria, C-11 acetate is incorporated into the tricarboxylic acid (TCA) cycle that generates electron donors for oxidative phosphorylation. Modeling of C-11 acetate myocardial retention has demonstrated that the first rapid phase of clearance correlates closely with oxygen consumption. ${ }^{27,28}$ Nony et al. evaluated TCA cycle activity and modelled myocardial oxygen consumption in six patients using $\mathrm{C} 11$ acetate clearance. ${ }^{18}$ Doxorubicin $\left(50 \mathrm{mg} / \mathrm{m}^{2}\right)$ did not acutely alter TCA activity (within 24 hours), nor did it following completion of the chemotherapy course, despite the fact that there was a mean $10 \%$ reduction in LVEF measured by RNA. The absence of an apparent effect on the TCA cycle does not mean that mitochondrial respiration is unaffected by doxorubicin. Importantly, oxidative phosphorylation was not assessed by this technique and disruption of the electron transport chain in mitochondria could still lead to the generation of reactive oxygen species and subsequent cellular toxicity.

\section{FUTURE PERSPECTIVES: HYBRID IMAGING WITH PET-MR}

There is an increasing role of cardiac MR in the evaluation of oncologic patients. MR provides high temporal and spatial resolution and the ability to measure LVEF with high accuracy and reproducibility. Measurement of myocardial inflammation and infiltrative processes with $\mathrm{T} 2$ and $\mathrm{T} 1$ and T2 mapping (e.g., myocarditis, amyloid, or iron deposition) serve as examples for which serial cardiac MR, LVEF and tissue characterization measures may be beneficial. ${ }^{29,30}$ In addition, CMR can perform both quantitative and semiquantitative assessments of myocardial perfusion reserve through contrasted stress examinations with agents such as adenosine. ${ }^{31}$ To date, however, cardiac MR measurements have not been readily adopted into position or guideline statements related to the management of patients receiving potentially cardiotoxic or renovascular-toxic cancer treatments. ${ }^{32}$

A combined technique with cardiac MR and PET to assess structural and functional responses to chemotherapy is inherently attractive and is increasing available with the advent of hybrid PET MR scanners. The promise of hybrid camera technology is that in future it will be possible to make correlations between changes in tracer kinetics and earlier structural changes detected at cardiac MR during the same sitting.

\section{CONCLUSIONS}

Susceptibility to cardiotoxicity from chemotherapy is currently difficult to determine. Identification of vulnerability is important to be able to monitor toxicity and adjust management accordingly. Adjustments must be timely since there is a relatively short time window for the recovery of cardiac function following cardiotoxicity. Laursen et al. have identified a potential earlier marker of the deleterious effects of anthracyclines on microvascular function through the use of $82-\mathrm{Rb}$ perfusion PET. They have shown there is a subset of patients with an abnormal response. The follow-up study from their cohort is awaited to determine the significance of their observations; for it remains uncertain where these observations lie on the proposed cardiotoxicity cascade (Figure 1). Whether reduced MBF is a subclinical marker of cardiotoxicity or a readily reversible sign of microvascular dysfunction will hopefully become clear with further investigation. If supported by such future studies, this technique could prove useful to assist decisions regarding the commencement or 
continuation of anthracycline therapy or the intensity of cardiac surveillance during or post chemotherapy. It is with optimistic but cautious anticipation, we await these data to support whether PET flow quantification can be considered useful in this clinical setting.

\section{Disclosure}

Dr. Ziadi has no disclosures.

\section{References}

1. Barac A, Murtagh G, Carver JR, et al. Cardiovascular health of patients with cancer and cancer survivors: a roadmap to the next level. J Am Coll Cardiol 2015;65:2739-46.

2. Mulrooney DA, Yeazel MW, Kawashima T, et al. Cardiac outcomes in a cohort of adult survivors of childhood and adolescent cancer: retrospective analysis of the Childhood Cancer Survivor Study cohort. BMJ 2009;339:4606.

3. Zhang S, Liu X, Bawa-Khalfe $\mathrm{T}$, et al. Identification of the molecular basis of doxorubicin-induced cardiotoxicity. Nat Med 2012;18:1639-42.

4. Volkova M, Russell R. Anthracycline cardiotoxicity: prevalence, pathogenesis and treatment. Curr Cardiol Rev 2011;7:21420.

5. Bristow MR, Thompson PD, Martin RP, Mason JW, Billingham ME, Harrison DC. Early anthracycline cardiotoxicity. Am J Med 1978;65:823-32

6. Dazzi H, Kaufmann K, Follath F. Anthracycline-induced acute cardiotoxicity in adults treated for leukaemia. Analysis of the clinico-pathological aspects of documented acute anthracycline induced cardiotoxicity in patients treated for acute leukaemia at the University Hospital of Zurich, Switzerland, between 1990 and 1996. Ann Oncol 2001;12:963-6.

7. Swain SM, Whaley FS, Ewer MS. Congestive heart failure in patients treated with doxorubicin a retrospective analysis of three trials. Cancer 2003;97:2869-79.

8. Armenian SH, Sun C, Shannon T, Mills G, Francisco L, Venkataraman $\mathrm{K}$, et al. Incidence and predictors of congestive heart failure after autologous hematopoietic cell transplantation. Blood 2011;118:6023-9.

9. Russell R, Alexander J, Schwartz RG, et al. The role and clinical effectiveness of multimodality imaging in the management of cardiac complications of cancer and cancer therapy. J Nucl Cardiol 2016.

10. Walker J, Bhullar N, Fallah-Rad N, et al. Role of three-dimensional echocardiography in breast cancer: comparison with twodimensional echocardiography, multiple-gated acquisition scans, and cardiac magnetic resonance imaging. J Clin Oncol 2010;28:3429-36.

11. Cardinale D, Colombo A, Bacchiani G, et al. Early detection of anthracycline cardiotoxicity an improvement with heart failure therapy. Circulation 2015;131:1981-8.

12. Raman SV, Shah M, McCarthy B, Garcia A, Ferketich AK. Multidetector row cardiac computed tomography accurately quantifies right and left ventricular size and function compared with cardiac magnetic resonance. Am Heart J 2006;151:736-44.

13. Jordan JH, Todd RM, Hundley G, et al. Cardiovascular magnetic resonance in the oncology patient. JACC Cardiovasc Imaging 2018;11:1150-72.
14. Marshall RC, Berger HJ, Reduto LA, Gottschalk A, Zaret BL. Variability in sequential measures of left ventricular performance assessed with radionuclide angiocardiography. Am J Cardiol 1978;41:531-6.

15. Schwartz RG, Jain D, Storozynsky E. Traditional and novel methods to assess and prevent chemotherapy-related cardiac dysfunction noninvasively. J Nucl Cardiol 2013;20:443-64.

16. De Geus-Oei LF, Mavinkurve-Groothuis AM, Bellersen L, et al. Scintigraphic techniques for early detection of cancer treatment induced cardiotoxicity. J Nucl Med Technol 2013;41:170-81.

17. Sarocchi M, Grossi F, Arboscello E, Spallarossa P, et al. Serial troponin for early detection of Nivolumab cardiotoxicity in advanced non-small cell lung cancer patients. Oncologist 2018;23:936-42.

18. Nony P, Guastalla JP, Rebattu P, Landais P, Lievre M, Bontemps L, Itti R, Beaune J, Andre-Fouet X, Janier M. In vivo measurement of myocardial oxidative metabolism and blood flow does not show changes in cancer patients undergoing doxorubicin therapy. Cancer Chemother Pharmacol 2000;45:375-80.

19. Laursen A, Elming M, Hasbak P, Thune J, et al. Rubidium- 82 positron emission tomography for detection of acute doxorubicininduced cardiac effects in lymphoma patients. J Nucl Cardiol 2018 , in press.

20. Ziadi MC, Beanlands RS. The clinical utility of assessing myocardial blood flow using positron emission tomography. J Nucl Cardiol 2010;17:571-81.

21. Parkash R, de Kemp RA, Ruddy TD, Beanlands RS, et al. Potential utility of rubidium 82 PET quantification in patients with 3-vessel coronary artery disease. J Nucl Cardiol 2004;11:440-9.

22. Murthy VL, Naya M, Foster CR, et al. Improved cardiac risk assessment with noninvasive measures of coronary flow reserve. Circulation 2011;124:2215-24.

23. Feola M, Chauvie S, Rosso GL, Biggi A, Ribichini F, Bobbio M. Reversible impairment of coronary flow reserve in takotsubo cardiomyopathy: a myocardial PET study. J Nucl Cardiol 2008; 15:811-7.

24. Schindler TH, Schelbert HR, Quercioli A, Dilsizian V. Cardiac PET imaging for the detection and monitoring of coronary artery disease and microvascular health. JACC Cardiovasc Imaging 2010;3:623-40.

25. Croteau E, Gascon S, Bentourkia M, Langlois R, Rousseau JA, Lecomte R, Bénard F. 11C Acetate rest-stress protocol to assess myocardial perfusion and oxygen consumption reserve in a model of congestive heart failure in rats. Nucl Med Biol 2012;39:287-94.

26. Bauckneht M, Massaia M, Gallamini A, Bruzzi P, Marini C, Sambuceti G. A score-based approach to 18F-FDG PET images as a tool to describe metabolic predictors of myocardial doxorubicin susceptibility. Diagnostics 2017. https://doi.org/10.3390/diagnos tics7040057.

27. Beanlands RS, Bach DS, Raylman R, Armstrong WF, Wilson V, Montieth M, Moore CK, Bates E, Schwaiger M. Acute effects of dobutamine on myocardial oxygen consumption and cardiac efficiency measured using carbon-11 acetate kinetics in patients with dilated cardiomyopathy. J Am Coll Cardiol 1993;22:1389-98.

28. Armbrecht JJ, Buxton DB, Schelbert HR. Validation of $11 \mathrm{C}$-acetate as a tracer for noninvasive assessment of oxidative metabolism with positron emission tomography in normal, ischemic, postischemic, and hyperemic canine myocardium. Circulation 1990;81:1594-605.

29. Friedrich MG, Sechtem U, Schulz-Menger J, et al. Cardiovascular magnetic resonance in myocarditis: a JACC white paper. J Am Coll Cardiol 2009;53:1475-87.

30. Moon JC, Messroghli DR, Kellman P, et al. Myocardial T1 mapping and extracellular volume quantification: a Society for 
Cardiovascular Magnetic Resonance (SCMR) and CMR Working Group of the European Society of Cardiology consensus statement. J Cardiovasc Magn Reson 2013;15:92.

31. Biglands JD, Magee DR, Sourbron SP, Plein S, Greenwood JP, Radjenovic A. Comparison of the diagnostic performance of four quantitative myocardial perfusion estimation methods used in cardiac MR imaging: CE-MARC substudy. Radiology 2015;275:393-402.
32. Jennifer H. Jordan, PHD, MS, Ryan M. Todd, BS, Sujethra Vasu, MBBS, W. Gregory Hundley, MD. Cardiovascular Imaging in the Oncologic Patient. JACC Cardiovasc Imaging 2018;11:1150-72.

Publisher's Note Springer Nature remains neutral with regard to jurisdictional claims in published maps and institutional affiliations. 